\title{
Analogues of nonlinear optics using terahertz Josephson plasma waves in layered superconductors
}

\author{
SERGEY SAVEL'EVV',, A. L. RAKHMANOV'1,3, V. A. YAMPOL'SKII 1,4 AND FRANCO NORII ${ }^{1,5 *}$ \\ ${ }^{1}$ Frontier Research System, The Institute of Physical and Chemical Research (RIKEN), Wako-shi, Saitama 351-0198, Japan \\ ${ }^{2}$ Department of Physics, Loughborough University, Loughborough LE11 3TU, UK \\ ${ }^{3}$ Institute for Theoretical and Applied Electrodynamics Russian Academy of Sciences, 125412 Moscow, Russia \\ ${ }^{4}$ A. Ya. Usikov Institute for Radiophysics and Electronics Ukrainian Academy of Sciences, 61085 Kharkov, Ukraine \\ ${ }^{5}$ Center for Theoretical Physics, Department of Physics, University of Michigan, Ann Arbor, Michigan 48109-1040, USA \\ *e-mail: nori@umich.edu
}

lectromagnetic waves in layered superconductors are known as Josephson plasma waves (JPWs). An important property of JPWs is the gap in their energy spectrum: JPWs can propagate if the frequency $\omega$ is above the Josephson plasma frequency $\omega_{J}$ (refs 1,2$)$, which being in the terahertz (THz) range, is important for applications ${ }^{3}$. This feature is fuelling a growing interest in studies of JPWs (see, for example, refs 4-7). However, nonlinear (NL) JPWs have not yet been studied. It is a challenge to understand nonlinearities around the plasma frequency, where the interplay between the unusual spectrum and the nonlinearity of the JPWs is most pronounced. Here, we predict the propagation of NL JPWs with frequencies below $\omega_{\mathrm{J}}$, which is unusual for plasma-like excitations. In analogy to NL optics, these waves exhibit numerous remarkable features, including the slowing down of light (when the group velocity $\partial \omega / \partial k \approx 0$ ), self-focusing effects and the pumping of weaker waves by stronger ones. The nonlinearity for $\omega>\omega_{\text {J }}$ can potentially be used for transforming continuous $\mathrm{THz}$ radiation into amplified pulses.

The nonlinear (NL) effects in optics are of both fundamental and technological interest ${ }^{8}$. They arise from the electric, $E$, or magnetic, $H$, field dependence of the refraction coefficient, $n$. The nonlinearity in Josephson media is due to the NL dependence, $j \propto \sin \varphi$, of the tunnelling supercurrent $j$ on the phase difference $\varphi$, which determines the fields $E$ and $H$.

This profound analogy between NL optics and NL JPW, summarized in Table 1, could open new avenues in the study of $\mathrm{THz}$ plasma waves in superconductors, providing a programme for future research in this growing field. Additional comparisons can be made with NL acoustics ${ }^{9}$, plasmas $^{10}$ and NL spin waves ${ }^{11}$. The control of $\mathrm{THz}$ radiation is important for applications in astronomy, chemistry, biology and medicine, including $\mathrm{THz}$ imaging, spectroscopy, tomography, medical diagnosis, health monitoring and environmental control, as well as chemical and biological identification.

Here, we predict (1) a propagating NL JPW below the Josephson plasma frequency when the JPW amplitude exceeds a critical value. Owing to damping related to the quasiparticle current, the NL JPW decays in the sample bulk and approaches the critical amplitude at which it cannot propagate. (2) At this amplitude, the group velocity of the JPWs approaches zero if the damping value $r$ tends to zero (that is, $\partial \omega / \partial k \propto r$ ). We also prove (3) that a localized beam of $\mathrm{NL} \mathrm{THz}$ radiation can propagate without spatial spreading below $\omega_{\mathrm{J}}$. This phenomenon is an analogue of the self-focusing effect in NL optics ${ }^{8}$. (4) A weak JPW that cannot penetrate the sample when $\omega<\omega_{\text {J }}$ can be assisted to propagate there by a NL JPW, in analogy with the self-induced transparency in NL optics. ${ }^{8}$. (5) At frequencies above $\omega_{\mathrm{J}}$, the nonlinearity produces a distortion of the resonance (which is due to the commensurability of the sample size with the JPW half length), including frequency hysteresis, in analogy with the resonance in anharmonic oscillators ${ }^{12}$. Our analytical results are supported by numerical simulations. Animations of these effects are available online, at http://dml.riken.jp/THz/nonlinear/nonlinear.swf. The considered NL phenomena in layered superconductors can be used to design a new generation of $\mathrm{THz}$ devices, including lenses and amplifiers. We also emphasize that our main equation (1) is not found in traditional NL optics.

\section{MODEL}

The gauge-invariant phase difference $\varphi$ in layered superconductors is described by a set of coupled sine-Gordon equations (see, for example, refs 13,14). So far, these equations have been studied for describing either Josephson vortices or linear waves. Here we focus on weakly $\mathrm{NL}\left(\sin \varphi \approx \varphi-\varphi^{3} / 6\right)$ waves at frequencies around $\omega_{\mathrm{J}}$ that, in the long-wavelength (compared with the interlayer spacing) limit, obey

$$
\left(1-\frac{\partial^{2}}{\partial y^{2}}\right)\left(\frac{\partial^{2} \varphi}{\partial t^{2}}+r \frac{\partial \varphi}{\partial t}+\varphi-\frac{\varphi^{3}}{6}\right)-\frac{\partial^{2} \varphi}{\partial x^{2}}=0 .
$$

Hereafter, we use dimensionless coordinates $x$ and $y$, and time $t$, $x \rightarrow x / \lambda_{c}, y \rightarrow y / \lambda_{a b}, t \rightarrow \omega_{\mathrm{J}} t, \omega \rightarrow \omega / \omega_{\mathrm{J}}$, where $\lambda_{a b}$ and $\lambda_{c}$ are the 


\begin{tabular}{|c|c|c|}
\hline & NL JPWs & Traditional NL optics \\
\hline Nonlinearity & Due to the NL current-phase relation $j=j_{c} \sin \varphi$ & $\begin{array}{l}\text { Due to, for example, NL refraction coefficient-electric } \\
\text { field relation } n(E)=n_{0}+n_{2} E^{2}\end{array}$ \\
\hline Higher harmonic frequencies generated from the basic $\omega$ & Only $3 \omega, 5 \omega, \ldots$ & $\begin{array}{l}2 \omega, \ldots, \text { (could be } 3 \omega, \text { or } 4 \omega \text { ) depending on the } \\
\text { nonlinearity }\end{array}$ \\
\hline Wave rectification and mixing & Yes & Yes \\
\hline Wave propagation below gap & Propagation of plane waves with $\omega<\omega_{\mathrm{J}}$ & - \\
\hline Slowing down EMW & THz wave can slow down significantly if $\omega<\omega_{\mathrm{J}}$ & Light can be slowed down \\
\hline Transparency due to nonlinearity & $\begin{array}{l}\text { Weak waves with } \omega<\omega_{\text {J }} \text {, which cannot originally propagate, } \\
\text { do propagate assisted by NL JPWs }\end{array}$ & Self-induced transparency \\
\hline NL pumping & Weak wave grows when the NL JPW is attenuated & Pumping of NL waves in plasma \\
\hline Focusing & Below $\omega_{\jmath}$, focused $\mathrm{THz}$ beam propagates & Self-focusing due to nonlinearity of $n(E)$ \\
\hline Wave packet spreading & Open problem & Can propagate without spreading \\
\hline Loading-unloading cycles due to nonlinearity & $\begin{array}{l}\text { Frequency hysteresis of NL geometric resonance converts } \\
\text { continuous radiation to amplified pulses } \\
\text { (analogy with NL mechanical resonance) }\end{array}$ & NL optical bistable devices \\
\hline
\end{tabular}

London penetration depths across and along the layers. The axes $x$ and $y$ are along and across the layers, whereas the $z$ axis is along the magnetic field of the NL JPWs. The dimensionless damping $r=4 \pi \sigma_{c} \lambda_{c} / c \ll 1$, where $\sigma_{c}$ is the $c$ axis quasiparticle conductivity, is controlled by the sample temperature $T, r \propto \exp (-\Delta / T)$, and can easily be reduced to negligibly small values, $r \ll 1$. As was shown in refs 15,16 , the intralayer quasiparticle conductivity, $\sigma_{a b}$, should also be included when $\omega$ is far enough from the plasma frequency. The contribution of the in-plane conductivity into the dissipation can easily be incorporated in our analysis. However, for the frequency range considered here (close to $\omega_{\text {J }},\left|1-\omega^{2}\right| \equiv$ $\left.\left|1-\left(\omega / \omega_{\mathrm{J}}\right)^{2}\right| \ll 1\right)$, this contribution is strongly suppressed and can safely be omitted because the relative value of the term with $\sigma_{a b}$ is $\left(1-\omega^{2}\right)\left(\sigma_{a b} / \sigma_{c}\right)\left(\lambda_{a b} / \lambda_{c}\right)^{2} \sim 10^{-3} \ll 1$. Here we used the standard values $\sigma_{a b} / \sigma_{c}=10^{5}, \lambda_{c} / \lambda_{a b}=500$, for Bi2212 compounds and consider $1-\omega \sim 10^{-3}$.

We study equation (1) using the asymptotic expansion

$$
\varphi=\sum_{n=0}^{\infty} a_{2 n+1}(x, y) \sin \left[(2 n+1) \omega t-\eta_{2 n+1}(x, y)\right],
$$

with spatially varying amplitude $a_{2 n+1}$ and phases $\eta_{2 n+1}$, to obtain periodic solutions. For waves with amplitude $a_{1} \sim \sqrt{\left|1-\omega^{2}\right|} \ll 1$, the NL term $\varphi^{3}$ in equation (1) is of the same order as the linear one, $\partial^{2} \varphi / \partial t^{2}+\varphi$, and a weak nonlinearity plays a key role in the wave propagation when the frequency $\approx \omega_{\mathrm{J}}$.

\section{NL PLANE WAVE BELOW THE PLASMA FREQUENCY}

For plane waves propagating along the $x$ axis, the asymptotic expansion of equation (1), with respect to $1-\omega^{2}$, produces a set of ordinary differential equations for $a_{1,3, \ldots}(x)$ and $\eta_{1,3, \ldots}(x)$ (see the Methods section, equation (6)). Owing to the nonlinearity, also described in Table 1, the propagating JPW includes higher harmonics with decreasing amplitudes $a_{2 n+1} \propto\left|1-\omega^{2}\right|^{n+1 / 2}$. At $r=0$, equation (6) has a solution with constant amplitudes and wavevectors $k$. For the first harmonics we derive

$$
\eta_{1}^{\prime}=k=\sqrt{\omega^{2}-1+a_{1}^{2} / 8} .
$$

From equations (2) and (3), we conclude that the NL JPW can propagate below $\omega_{\mathrm{J}}$, if its amplitude is strong enough: $a_{1}^{2}>a_{c}^{2}=8\left(1-\omega^{2}\right)$. This result (confirmed by numerical simulations, for example, in Fig. 1) is very unusual for any conducting media, where plasma waves propagate only with frequencies above the plasma resonance. Wave packets formed by superpositions of NL JP plane waves exhibit weak spreading if their frequency widths are much less than $1-\omega$. Using the Maxwell and Josephson equations, we find that, at $a_{1}=a_{c}$, the amplitude of the magnetic field in the running wave is $H_{0 c}=H_{0} a_{c}^{2}$; where $H_{0}=\Phi_{0} / 2 \pi s \lambda_{c}$, where $s$ is the period of the superconducting layered structure and $\Phi_{0}$ is the flux quantum. For layered superconductors, $H_{0} \sim 20$ Oe. Assuming $1-\omega=10^{-3}$, we find $H_{0 c}=0.32 \mathrm{Oe}$. If the magnetic field amplitude of the incident and running waves are of the same order, then the power necessary to excite NL JPWs should be larger than $0.25 \mathrm{~W} \mathrm{~cm}^{-2}$. This power entering the sample might be much lower than the incident power.

These NL waves can be excited by applying $\mathrm{THz}$ radiation to the sample edge using the experimental setup described, for example, in ref. 17. The propagation of NL JPW could be detected by measuring the reflection coefficient or the shift of the plasma resonance as a function of the applied amplitude of the electromagnetic field.

\section{SLOWING DOWN OF LIGHT}

Dissipation $(r \neq 0)$ produces wave damping, and $a_{1}$ decays with $x$. At some $x=x_{0}$, the amplitude $a_{1}(x)$ achieves the critical value $a_{c}$. At this point, the wavevector $k$ and the group velocity $v_{\mathrm{g}}=\partial \omega / \partial k \propto$ $\sqrt{a_{1}-a_{c}}$ vanish according to equation (3). In other words, the 'stopping-of-light' phenomenon occurs. A more-detailed analysis yields an estimation for the minimum $v_{\mathrm{g}}: v_{\mathrm{g}}^{\min } \sim r / \sqrt{1-\omega^{2}}$, if $\left(1-\omega^{2}\right)^{2} \ll r \ll\left(1-\omega^{2}\right) ; v_{\mathrm{g}}^{\min } \sim\left(1-\omega^{2}\right)^{3 / 2}$, if $r \ll\left(1-\omega^{2}\right)^{2}$. For example, at $r=10^{-6}, 1-\omega=10^{-3}, \lambda_{c}=10^{-2} \mathrm{~cm}, \omega_{\mathrm{J}}=10^{11} \mathrm{~s}^{-1}$, we find that $v_{\mathrm{g}}^{\min } \sim 10^{5} \mathrm{~cm} \mathrm{~s}^{-1}$. The stopping-of-light phenomenon has possible applications for quantum information processing and the artificial creation of 'event horizons ${ }^{18,19}$ in solids. Far from $x=x_{0}$ (deeper in the sample), the nonlinearity becomes irrelevant and the JPW decays on a scale $\sim 1 / \sqrt{1-\omega^{2}}$. The Josephson current is cancelled by the displacement current at $x \sim x_{0}$, where the stoplight phenomenon occurs. As a result, there is a standing wave with small $H$ compared with the electric field $E$ in this part of the sample. This standing wave can be observed by low-temperature scanning (either electron ${ }^{20}$ or laser $^{21}$ ) microscopy. 


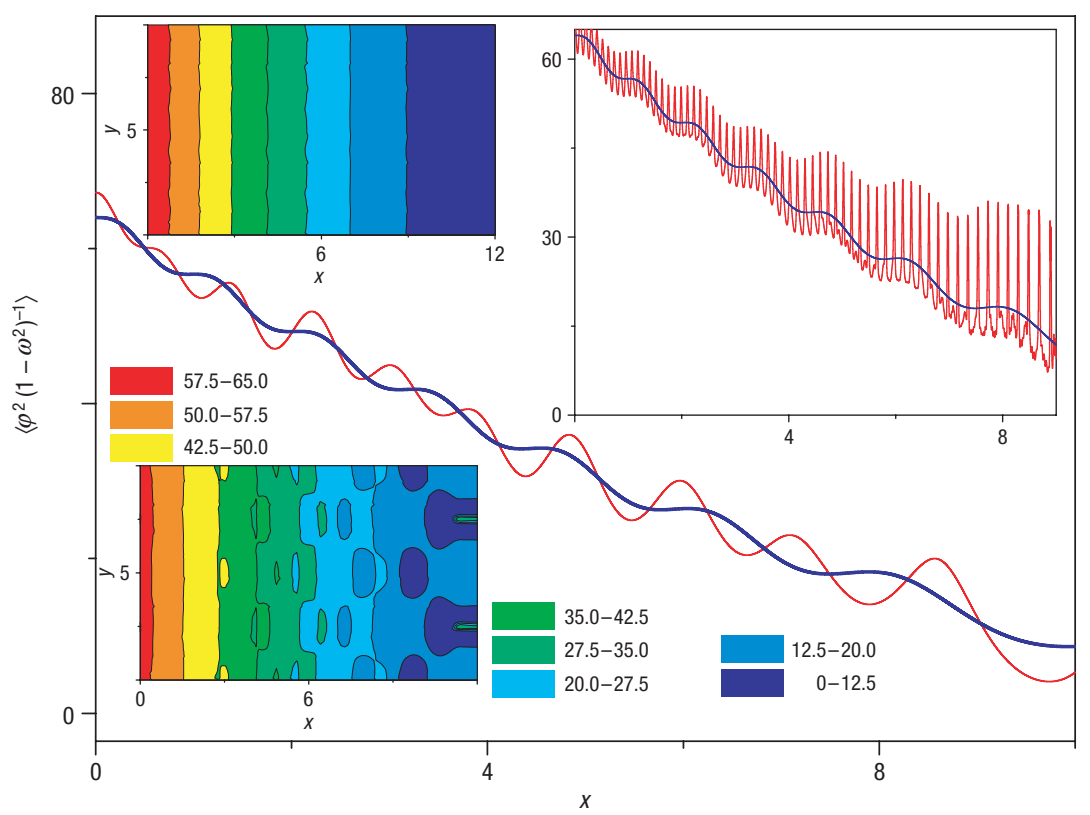

Figure 1 Self-induced transparency and NL pumping. Numerically obtained self-induced transparency and pumping: the spatial dependence of the normalized time average of $\varphi^{2}(x, y=0)$ for a single NL JPW with $a_{1}(0)=8 \sqrt{\left(1-\omega^{2}\right)}, r=0.5$ (blue line) and for a mixture (red line) of NL JPWs with the same parameters and a weaker wave with amplitude $0.2 \cos (q y)$ at the surface. Here, $q=0.4 \pi(q=4 \pi)$ for the red line in the main panel (right top inset). Left top (bottom) inset shows a $2 \mathrm{D}$ contour plot for the blue (red) line in the main panel.

\section{SELF-INDUCED TRANSPARENCY}

We show that the NL plane wave with $\omega<1$ is stable with respect to small fluctuations $\delta \varphi \propto \exp (i q y+i p x-i \omega t)$ : the dispersion equation for $p$ has only real solutions. For example, at $r=0$, $p= \pm \sqrt{\left(1+q^{2}\right)\left[2\left(1-\omega^{2}\right)+3 k^{2}\right]-k^{2}}$. This indicates that the NL wave assists the propagation of linear waves of the same frequency and wavevector $p$, which could not propagate by themselves because $\omega<1$. This effect is the JPW analogue of the self-induced transparency in NL optics (see Table 1).

\section{NL PUMPING OF A WEAK WAVE BY A STRONG ONE}

We have shown above that a running NL wave allows propagation of weak waves below the plasma frequency $\omega_{\mathrm{J}}$. More interestingly, we have shown that a decaying NL wave, with amplitude $a_{1}$ below a critical value $a_{c}$, pumps weak waves with large transverse wavenumber $q$. This occurs (see the Methods section) if either the amplitude of the NL wave drops below $a_{c}$ due to dissipation, or the amplitude of the incident wave is below the propagating threshold.

\section{NUMERICAL SIMULATIONS}

To test the validity of our analytical results briefly summarized above, we carry out numerical simulations of equation (1), shown in Fig. 1, for: (1) JPWs propagating below $\omega_{\mathrm{J}}$, (2) self-induced transparency and (3) pumping. NL JPWs with $\omega<1$ propagate inside the sample, weakly decaying due to damping (blue line in the main panel and 2D plot in the top left inset). A weak wave with $\omega<1$ cannot propagate alone. However, the strong JPW assists the propagation and pumps a weaker wave (red line in main panel, 2D plot in the bottom left inset and top right inset). Stronger pumping occurs for higher values of $q$.
We now describe a possible experiment to observe self-induced transparency and NL pumping. We propose applying a continuous weak electromagnetic wave (EMW) with $k, q \neq 0$ to a sample surface parallel to the $a b$ plane, and also pulses of strong radiation to a sample edge. Then sweeping the incident angle $\theta$ of the weak signal, a significant change in the reflectivity and transmissivity coefficients should be observed at a certain $\theta$, when the strong wave is switched on. This effect could be useful for THz filters.

\section{LOCALIZED THz BEAM}

Now we focus on 'beam solutions', $\varphi=a_{1}(y) \sin (\omega t-k x)$, of equation (1) for NL waves with $\omega<1$, and $a_{1}=0$ at $y \rightarrow \pm \infty$. If we neglect the dissipation $(r=0)$ and higher-harmonic generation, the amplitude $a_{1}(y)$ satisfies

$$
\left(1-\frac{\mathrm{d}^{2}}{\mathrm{~d} y^{2}}\right)\left[\left(1-\omega^{2}\right) a_{1}-\frac{a_{1}^{3}}{8}\right]+k^{2} a_{1}=0
$$

with boundary conditions $a_{1}( \pm \infty)=0$. This equation has an analytical solution that has a beam structure, that is, localized in the $y$ direction. The magnetic field distribution in the beam is shown in Fig. 2. This self-sustained solution is an analogue of the selffocusing effect in NL optics. This beam could be directly excited by applying, from the sample edge, magnetic field radiation with a profile similar to that in Fig. 2.

\section{NL GEOMETRIC RESONANCE}

Now we consider an EMW, $H=H_{\mathrm{i}} \mathrm{e}^{i k_{0} x-i \omega t}+H_{\mathrm{r}} \mathrm{e}^{-i k_{0} x-i \omega t}$, with frequency above $\omega_{\mathrm{J}}$, incident from the vacuum on a slab sample $-l<x<l$. Here $H_{\mathrm{i}}$ and $H_{\mathrm{r}}$ are the amplitudes of the incident and reflected waves, respectively. In linear approximation 


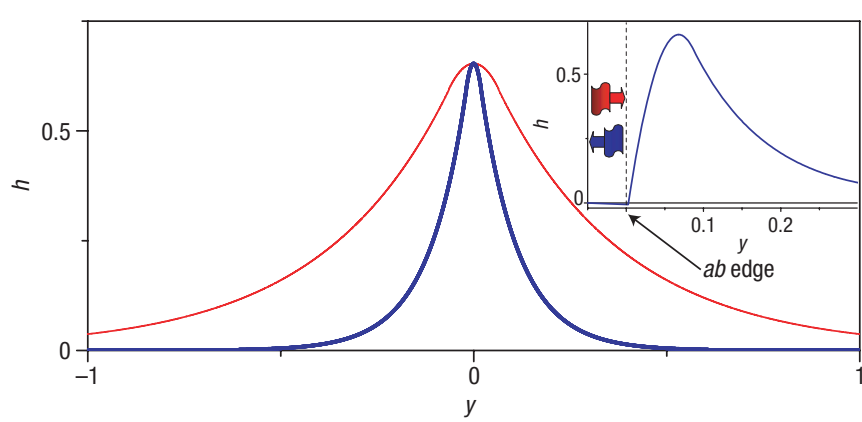

Figure 2 Focused THz beam and NL surface wave. The normalized magnetic field $h=H / \mathscr{H}, \mathscr{H}=H_{0}\left(1-\omega^{2}\right)^{3 / 2} / k$, versus the transverse coordinate $y$ for different wavenumbers: $k\left(1-\omega^{2}\right)^{-1 / 2}=3$ (red) or 10 (blue) lines. Inset: the normalized magnetic field in the NL surface wave excited by a THz wave (shown by a red arrow) incident on the large $\left(\sim 1 \mathrm{~mm}^{2}\right)$ top surface parallel to the $a b$ plane; same parameters as for the localized THz beam shown by the blue line in the main panel. The reflected wave (shown by the blue arrow) can be strongly suppressed because of the resonant excitation (that is, Wood's anomalies) of the NL surface waves.

theory, the incident EMW excites a wave in the sample $\varphi=a^{+} \mathrm{e}^{i k x-i \omega t}+a^{-} \mathrm{e}^{-i k x-i \omega t}$. To find the amplitudes $a^{ \pm}$, we use the continuity of the magnetic field and the tangential component of the electric field at the vacuum-sample interface. If a half length of the EMW is commensurate with the sample length, $k l=\pi n / 2$, the reflected wave in the vacuum disappears, the sample becomes completely transparent ${ }^{5}$, and the amplitudes of the EMW in the sample increase. Near this resonance at small $r \ll 1$ and $a^{ \pm} \ll 1$ (when the expansion $\sin \varphi \approx \varphi-\varphi^{3} / 6$ is valid), the amplitudes $a^{ \pm}$ are defined by

$$
a^{+}=a^{-}=\frac{H_{\mathrm{i}}}{H_{0}} \frac{Q \sqrt{\varepsilon} \mathrm{e}^{-i k_{0} l}}{2 \omega(1+i Q l \delta k)},
$$

where $\delta k=k-\pi n / 2 l, k_{0}=\omega / \sqrt{\varepsilon}, \varepsilon$ is the interlayer dielectric constant, and $Q=2 l k_{0} / \pi n$. According to equation (5), the energy density stored in the sample near the resonance exceeds the EMW energy density in vacuum by the factor $\mathcal{K}=\omega^{2} l^{2} / \pi^{2} n^{2} \varepsilon$. Below we consider only the case $n=1$ and $\omega \approx 1$ (that is, close to plasma frequency), where the effect is most pronounced. Taking, as an estimate, the sample length $2 l \lambda_{c}=2 \mathrm{~cm}, \lambda_{c}=10^{-2} \mathrm{~cm}$, and $\varepsilon=10$, we obtain $\mathcal{K} \approx 100$.

The influence of the nonlinearity on the resonance can be analysed assuming the dependence of $k$ on the wave amplitude, in analogy to the well-known NL mechanical resonance $^{12}$. The dependence of $k$ on the amplitudes $a^{ \pm}$is similar to equation (3) when replacing $a_{1}^{2} / 8$ by $3\left|a^{+}\right|^{2} / 8$. Thus, $\delta k=2 l\left(3\left|a^{+}\right|^{2} / 16+\Delta \omega\right) / \pi$, where $\Delta \omega$ is the detuning of the EMW frequency from the resonance value $\omega_{\text {res }}=\sqrt{1-\pi^{2} / 4 l^{2}}$. Substituting $\delta k$ in equation (5), we derive a cubic equation for $\left|a^{+}\right|^{2}$. Solving this equation we find the dependence of the wave amplitude on the frequency near the resonance. For a small amplitude of the incident wave, $H_{\mathrm{i}}$, the resonance peak is slightly distorted due to nonlinearity (Fig. 3). If $H_{\mathrm{i}}$ exceeds a threshold value, $H_{\text {thr }} \approx 7.8 H_{0} \varepsilon^{1 / 4} / l^{5 / 2}$, two different stable solutions with higher and lower amplitudes $\left|a^{+}\right|$occur near the resonance. In analogy with NL mechanical resonance ${ }^{12}$, this produces a hysteretic behaviour of $a^{+}(\omega)$ when slowly sweeping the frequency $\omega$ of the incident wave (see Fig. 3 and Table 1). As a result, the abrupt transitions between two solutions and, thus, an almost immediate release of the accumulated energy, occurs at $\Delta \omega=\omega_{c} \approx 4.3 \sqrt{\varepsilon} / l^{3}$.

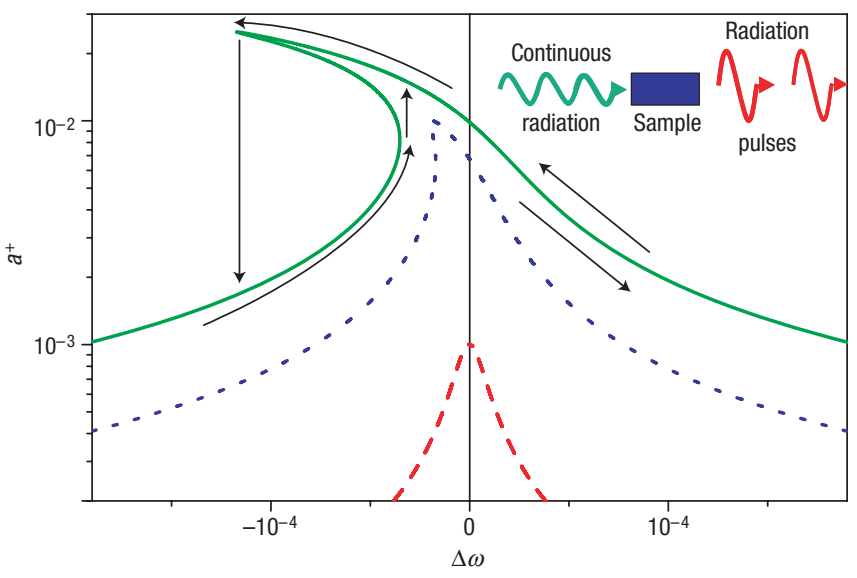

Figure $3 \mathrm{NL}$ geometric resonance and THz wave amplification. The amplitude $\mathrm{a}^{+}$ of a wave inside the sample versus detuning frequency $\Delta \omega$ for different incident wave amplitudes: the red dashed line corresponds to $H_{\mathrm{i}} / H_{0}=10^{-5}$, the blue dotted line to $H_{\mathrm{i}} / H_{0}=10^{-4}$, and the green solid line to $H_{\mathrm{i}} / H_{0}=2.5 \times 10^{-4}$. Inset: schematic diagram showing the conversion of continuous $\mathrm{THz}$ radiation into amplified pulses.

Using the same parameters as above and $s=1.5 \mathrm{~nm}$, we estimate $H_{\mathrm{thr}} \approx 3 \times 10^{-3}$ Oe and $\Delta \omega \approx 1.4 \times 10^{-5} \omega_{\mathrm{J}}$.

We propose an experiment to observe this NL resonance. When a continuous EMW with time-dependent frequency $\omega=\omega_{\text {res }}+$ $\alpha \cos \left(\omega_{1} t\right)$, with $\alpha, \omega_{1} \ll \omega_{\text {res }}$, is applied to the sample edge ${ }^{17}$, an almost standing wave is excited in the layered superconductor. When $\alpha \cos \left(\omega_{1} t_{n}\right)=\omega_{c}$, this standing wave is periodically collapsed producing strong pulses of $\mathrm{THz}$ waves, which could be measured. Thus, such a device (inset in Fig. 3) could be used as an amplifier or as a converter of continuous $\mathrm{THz}$ radiation into short $\mathrm{THz}$ pulses (see also at http://dml.riken.jp/THz/nonlinear/nonlinear.swf).

\section{COUPLING NL WAVES WITH THE THZ FIELD IN THE VACUUM}

For anisotropic cuprate single crystals, the edge height along the $c$ axis direction is about $10-100 \mu \mathrm{m}$, whereas the $\mathrm{THz}$ wavelength in the vacuum is about $300 \mu \mathrm{m}$. Thus, the focusing of incident $\mathrm{THz}$ waves on the edge surface could be hard to achieve. Shining the top of the sample, with a surface of several $\mathrm{mm}^{2}$, at a small incident angle, avoids this problem. For instance, when the amplitude of the incident wave is large enough $\left(H_{0}>H_{0 c}\right)$, NL surface waves with a localized beam profile similar to Fig. 2 can be excited. The inset of Fig. 2 shows the field distribution in such a NL wave, obtained by solving equation (4) and matching the impedance $\left(E_{x} / H\right)$ on the vacuum-superconductor interface.

\section{METHODS}

\section{ASYMPTOTIC EXPANSION}

Substituting (2) into equation (1) we obtain (in the particular case studied above) the set of ordinary differential equations for harmonic amplitudes:

$$
\begin{gathered}
a_{1}^{\prime \prime}-\left[1-\omega^{2}+\left(\eta_{1}^{\prime}\right)^{2}\right] a_{1}+a_{1}^{3} / 8=0, \\
r \omega a_{1}+2 a_{1}^{\prime} \eta_{1}^{\prime}+a_{1} \eta_{1}^{\prime \prime}=0, \\
a_{3}^{\prime \prime}-\left[1-9 \omega^{2}+\left(\eta_{3}^{\prime}\right)^{2}\right] a_{3}+a_{1}^{3} \cos \left(\eta_{3}-3 \eta_{1}\right) / 24=0, \\
3 r \omega a_{1}+2 a_{3}^{\prime} \eta_{3}^{\prime}+a_{3} \eta_{3}^{\prime \prime}+a_{1}^{3} \sin \left(\eta_{3}-3 \eta_{1}\right) / 24=0,
\end{gathered}
$$

used to derive a spectrum of NL plane waves. 


\section{NL WAVE PUMPING}

For $a_{1}<a_{c}=\left[8\left(1-\omega^{2}\right)\right]^{1 / 2}$, the strong wave, $\varphi(x, t) \approx a_{1}(x) \sin \omega t$, decays on a scale $1 / \sqrt{1-\omega^{2}}$. A weak wave, $\varphi_{q}=a_{q}(x) \mathrm{e}^{i q y} \sin (\omega t)$, interacting with the strong one is described by the equation

$$
a_{q}^{\prime \prime}+\left(1+q^{2}\right)\left[\frac{3 a_{1}^{2}(x)}{8}-1+\omega^{2}\right] a_{q}=0,
$$

derived from equation (1). It is seen that at $a_{1}^{2}(x)>8\left(1-\omega^{2}\right) / 3$, the last equation describes a non-decaying wave. In the Wentzel-Kramers-Brillouin approximation, for $q \gg 1$, we find from equation (7)

$$
\frac{a_{q}(x)}{a_{q}(0)}=\left[\frac{3 a_{1}^{2}(0)-8\left(1-\omega^{2}\right)}{3 a_{1}^{2}(x)-8\left(1-\omega^{2}\right)}\right]^{1 / 2} \cos \left[\sqrt{1+q^{2}} \int_{0}^{x} \mathrm{~d} x^{\prime} \sqrt{\omega^{2}-1+3 a_{1}^{2}\left(x^{\prime}\right) / 8}\right] .
$$

The amplitude of the weak wave increases as $a_{1}(x)$ approaches a 'turning point' $x_{1}$, where $a_{1}^{2}\left(x_{1}\right)=8\left(1-\omega^{2}\right) / 3$. This indicates the pumping of weak waves with short $c$ axis wavelength.

\section{ASYMPTOTIC BEHAVIOUR OF NL THZ BEAM}

The analytical solution for a localized beam when $k^{2} \gg 1-\omega^{2}$ behaves as:

$$
\begin{gathered}
a_{1} \propto \exp \left(-\frac{k|y|}{\sqrt{\left(1-\omega^{2}\right)}}\right), \quad|y| \gg y_{0}, \\
a_{1} \propto \sqrt{\left(1-\omega^{2}\right)}\left(1-\frac{k^{2} y^{2}}{2\left(1-\omega^{2}\right)}\right), \quad|y| \ll y_{0},
\end{gathered}
$$

where $y_{0}=\sqrt{\left(1-\omega^{2}\right)} / k$. Far from the centre of the beam, $|y| \gg y_{0}$, the nonlinearity is not important but the wave is self-sustained because of its positive curvature, $\mathrm{d}^{2} a_{1} / \mathrm{d} y^{2}>0$. Indeed, the standard linear spectrum following from equation (1) is $k^{2}=-\left(1+q^{2}\right)\left(1-\omega^{2}\right)$. If $q^{2}>0$, the wave cannot propagate because $k^{2}<0$ when $\omega<1$; in contrast, for the beam, $q^{2} \approx-1 /\left(k^{2}+1-\omega^{2}\right)<0$, and the wavevector now satisfies the propagation condition, $k^{2}>0$, from the dispersion relation $k(q, \omega)$. Near the centre of the beam, nonlinearity joins two peripheral symmetric solutions with positive curvature allowing a self-sustained beam propagation. There are two points, $y \sim \pm y_{0}$, where the phase difference $\varphi$ exhibits a jump, whereas the magnetic field is continuous. As a result, the breaking of the charge neutrality occurs at the phase jumps.
Received 25 December 2005; accepted 14 June 2006; published 9 July 2006.

\section{References}

1. Bulaevskii, L. N., Maley, M. P. \& Tachiki, M. Low frequency magneto-optical properties of Josephson-coupled superconductors. Phys. Rev. Lett. 74, 801-804 (1995).

2. Matsuda, Y., Gaifullin, M. B., Kumagai, K., Kadowaki, K. \& Mochiku, T. Collective Josephson plasma resonance in the vortex state of Bi2212. Phys. Rev. Lett. 75, 4512-4515 (1995).

3. Phil. Trans. R. Soc. A 362 (special issue), 197-414 (2004).

4. Helm, Ch. \& Bulaevskii, L. N. Optical properties of layered superconductors near the Josephson plasma resonance. Phys. Rev. B 66, 094514 (2002).

5. Savel'ev, S., Rakhmanov, A. L. \& Nori, F. Using Josephson vortex lattices to control terahertz radiation: Tunable transparency and terahertz photonic crystals. Phys. Rev. Lett. 94, 157004 (2005).

6. Savel'ev, S., Yampol'skii, V. \& Nori, F. Surface Josephson plasma waves in layered superconductors. Phys. Rev. Lett. 95, 187002 (2005).

7. Savel'ev, S., Yampol'skii, V., Rakhmanov, A. \& Nori, F. Generation of tunable terahertz out-of-plane radiation using Josephson vortices in modulated layered superconductors. Phys. Rev. B 72, 144515 (2005).

8. Mills, D. L. Nonlinear Optics (Springer, Berlin, 1998).

9. Hamilton, M. F. \& Blackstock, D. T. (eds) in Nonlinear Acoustics: Theory and Applications (Academic, New York, 1998)

10. Horton, C. W. \& Ichikawa, Y. H. I. H. Chaos and Structures in Nonlinear Plasmas (World Scientific, Singapore, 1996).

11. Cottam, M. G. (ed.) Linear and Nonlinear Spin Waves in Magnetic Films and Superlattices (World Scientific, Singapore, 1994)

12. Landau, L. D. \& Lifshitz, E. M. Mechanics (Butterworth-Heinemann, Oxford, 1995).

13. Sakai, S., Bodin, P. \& Pedersen, N. F. Fluxons in thin-film superconductor-insulator superlattices. J. Appl. Phys. 73, 2411-2418 (1993).

14. Artemenko, S. N. \& Remizov, S. V. Stability, collective modes and radiation from sliding Josephson vortex lattice in layered superconductors. Physica C 362, 200-204 (2001).

15. Koshelev, A. E. Role of in-plane dissipation in dynamics of a Josephson vortex lattice in high-temperature superconductors. Phys. Rev. B 62, R3616-R3619 (2000).

16. Latyshev, Yu. L., Koshelev, A. E. \& Bulaevskii, L. N. Probing quasiparticle dynamics in Bi2212 with a driven Josephson vortex lattice. Phys. Rev. B 68, 134504 (2003).

17. Yamada, Ya. et al. Shapiro step responses in the flux-flow state of Bi2212 intrinsic Josephson junctions with cooperation of pancake vortices. IEEE Trans. Appl. Supercond. 15, 1028-1031 (2005).

18. Hawking, S. \& Penrose, R. The Nature of Space and Time (Princeton Univ. Press, Princeton, 2000).

19. Weinberg, S. Gravitation and Cosmology: Principles and Applications of the General Theory of Relativity (Wiley, New York, 1972).

20. Claus, T. et al. Imaging of $\mu \mathrm{m}$ wavelength collective cavity resonances in Bi2212 intrinsic Josephson junction stacks under microwave irradiation. Appl. Phys. Lett. 85, 3166-3168 (2004).

21. Sivakov, A. G. et al. Low-temperature scanning laser microscopy of individual filaments extracted from $(\mathrm{Bi}, \mathrm{Pb})_{2} \mathrm{Sr}_{2} \mathrm{Ca}_{2} \mathrm{Cu}_{3} \mathrm{O}_{10+x}$ tapes. Appl. Phys. Lett. 76, 2597-2599 (2000).

\section{Acknowledgements}

This work was supported in part by the NSA and ARDA under AFOSR contract No. F49620-02-1-0334, by the NSF grant No. EIA-0130383, the JSPS-RFBR project No 06-02-91200, the RFBR project 06-02-16691, and by an EPSRC Advanced Research Fellowship.

Correspondence and requests for materials should be addressed to F.N

Competing financial interests

The authors declare that they have no competing financial interests.

Reprints and permission information is available online at http://npg.nature.com/reprintsandpermissions/ 